\title{
Publisher Correction: Common carp aquaculture in Neolithic China dates back 8,000 years
}

Tsuneo Nakajima D, Mark J. Hudson, Junzo Uchiyama, Keisuke Makibayashi and Juzhong Zhang

Correction to: Nature Ecology \& Evolution https://doi.org/10.1038/s41559-019-0974-3, published online 16 September 2019.

In the version of this Brief Communication originally published, in the legend above the graph in Fig. 1i the descriptions for the white and grey data were inadvertently switched; they should have read "Cyprinus (black), Carassius (white), others (grey)"; this has now been corrected. 